\title{
Establishing a Methodology for Ultrasound Evaluation of Pharyngeal Residue in the Pyriform Sinus and Epiglottic Vallecula
}

\author{
Yuka Miura, Koichi Yabunaka, Mikihiko Karube, Takuya Tsutaoka, Mikako Yoshida, \\ Masaru Matsumoto, Gojiro Nakagami, Yayoi Kamakura, Junko Sugama, and Hiromi Sanada
}

\begin{abstract}
BACKGROUND: Assessing the presence of pharyngeal residue in the pyriform sinus and epiglottic vallecula is important because insufficient pharyngeal clearance is a risk factor for aspiration pneumonia. Improvements in the performance of ultrasound to visualize the pyriform sinus and epiglottic vallecula are needed. The aim of this study was to establish a method to visualize the pyriform sinus and epiglottic vallecula with ultrasound to detect pharyngeal residue. METHODS: We used real-time virtual sonography (ie, a fusion of magnetic resonance imaging and ultrasound imaging) as the scanning method to visualize the pyriform sinus and epiglottic vallecula without residue in $\mathbf{4}$ healthy individuals. Using established ultrasound methodology and fiberoptic endoscopic evaluation of swallowing, 35 subjects with dysphagia were studied to investigate the performance of ultrasound to detect pharyngeal residue. RESULTS: The fusion ultrasound images showed that transverse scans at the level of the laryngeal prominence and above the hyoid bone using a linear array transducer can be used to visualize the pyriform sinus and the epiglottic vallecula, respectively. We obtained 238 ultrasound images of the pyriform sinus from 35 subjects and 82 images of epiglottic vallecula from 26 of 35 subjects. The ultrasound images with fiberoptic endoscopic evaluation of swallowing showed that areas of high echogenicity in the pyriform sinus and epiglottic vallecula are related to the presence of pharyngeal residue. The presence of high-echogenicity areas resulted in a sensitivity of $\mathbf{9 2 . 0 \%}$ and specificity of $71.9 \%$ for detecting pharyngeal residue in the pyriform sinus and a sensitivity of $86.7 \%$ and specificity of $63.6 \%$ for detecting pharyngeal residue in the epiglottic vallecula. CONCLUSIONS: Transverse ultrasound scans at the level of the laryngeal prominence and above the hyoid bone enable the visualization of the pyriform sinus, epiglottic vallecula, and pharyngeal residue. Key words: aspiration pneumonia; swallowing disorders; pharynx; residue; secretion management; ultrasonography. [Respir Care 2020;65(3):304-313. (C) 2020 Daedalus Enterprises]
\end{abstract}

\section{Introduction}

Pharyngeal residue is defined as the presence of food, liquid, and secretion residues that are not cleared by swal-

\footnotetext{
Drs Miura and Matsumoto, as well as Mr Karube and Mr Tsutaoka, are affiliated with the Department of Imaging Nursing Science, Graduate School of Medicine, The University of Tokyo, Tokyo, Japan. Dr Yabunaka is affliated with Ohno Memorial Hospital, Osaka, Japan. Dr Yoshida is affiliated with Department of Women's Health Nursing \& Midwifery, Tohoku University Graduate school of Medicine, Miyagi, Japan. Drs Nakagami and Sanada are affiliatd with the Global Nursing Research Center, Graduate School of Medicine, The University of Tokyo, Tokyo, Japan. Mr Karube and Mr Tsutaoka are affiliated with the Imaging Technology Center, Research \& Development Management Headquarters, Fujifilm Corporation, Tokyo, Japan. Drs Nakagami and Sanada are affiliated with the Department of Gerontological Nursing/Wound Care Management, The University of Tokyo,
}

lowing. ${ }^{1}$ People with dysphagia often show insufficient pharyngeal clearance with pharyngeal residue due to impairment of the driving forces of the oropharyngeal bolus and reduced swallowing efficiency. ${ }^{2}$ Moreover, insufficient pharyngeal clearance is one of the risk factors for aspiration pneumonia because the residue sometimes moves into the airway in the absence of a cough reflex in people

\footnotetext{
Tokyo, Japan. Dr Kamakura is affiliated with the Japanese Red Cross Toyota College of Nursing, Aichi, Japan. Dr Sugama is affiliated with the Institute for Frontier Science Initiative, Kanazawa University, Ishikawa, Japan.

The authors have received support from the Japan Agency for Medical Research and Development. Drs Miura and Matsumoto, as well as $\mathrm{Mr}$ Karube and Mr Tsutaoka, have disclosed a relationship with Fujifilm Corporation. The other authors have disclosed no conflicts of interest.
} 
with dysphagia. ${ }^{3}$ The presence of pharyngeal residue increases the risk of aspiration $>2.8$ times compared with the risk in individuals without pharyngeal residue. ${ }^{4}$ The annual number of cases of aspiration pneumonia in Japan (the most aged society in the world) was estimated in 2013 to be $630,000 .{ }^{5}$ Effective pharyngeal clearance management to prevent aspiration pneumonia is needed in the aging population. Modifications of an individual's posture and of the volume and viscosity of foods and liquids have been shown to facilitate pharyngeal clearance. ${ }^{6,7}$ Moreover, it has been reported that pharyngeal residue suctioning by nurses can prevent aspiration pneumonia in subjects with acute stroke. ${ }^{8}$ Thus, the detection of pharyngeal residue and the management of pharyngeal clearance by nurses providing swallowing and secretion care will be effective strategies for aspiration pneumonia prevention.

Most pharyngeal residue is present in the pyriform sinus and the epiglottic vallecula. ${ }^{9}$ The pyriform sinus is in the hypopharyngeal area, and the epiglottic vallecula is the space between the base of the tongue and the epiglottis. These areas are difficult to observe directly by physical examination. Fiberoptic endoscopic evaluation of swallowing (FEES) is recognized as a sensitive tool for detection of pharyngeal residue in the pyriform sinus and the epiglottic vallecula. ${ }^{10}$ However, some individuals with dysphagia cannot tolerate FEES, which requires the insertion of a scope in the nose. Detecting abnormal sounds by cervical auscultation is a less invasive method than FEES, but a provider must be highly trained to be able to interpret the sounds or to perform complicated acoustic analyses. ${ }^{11,12}$ Thus, a less invasive imaging tool to visualize the pyriform sinus and epiglottic vallecula in real time would assist care professionals, including nurses, in removing the residue, and it would help prevent aspiration pneumonia.

Ultrasound imaging is a noninvasive, real-time assessment tool that has been applied for airway assessments that include the hypopharyngeal area. ${ }^{13,14}$ Few studies have focused on pharyngeal residue detection by ultrasound examination. A study showed a sensitivity of $62 \%$ and specificity was $67 \%$ for ultrasound examinations of pharyngeal residue $^{15}$ using ultrasound images that were originally intended to detect aspiration. ${ }^{16}$ The scanning method to detect aspiration focuses on the tracheal area, but the method to detect pharyngeal residue should focus instead on the pyriform sinus and epiglottic vallecula. Moreover, we needed to identify anatomical landmarks to systematize a methodology for ultrasound evaluation of pharyngeal residue both in the pyriform sinus and epiglottic vallecula.

Correspondence: Hiromi Sanada PhD RN, 7-3-1, Hongo, Bunkyo-ku, Tokyo, 113-0033, Japan. E-mail: hsanada-tky@umin.ac.jp.

DOI: $10.4187 /$ respcare.07002

\section{QUICK LOOK}

\section{Current knowledge}

Pharyngeal residues in the pyriform sinus and epiglottic vallecula cause aspiration pneumonia among individuals with dysphagia. Ultrasound examination has been used for airway assessment of the pyriform sinus and epiglottic vallecula, but the established method published for pharyngeal residue in the pyriform sinus and epiglottic vallecula has low sensitivity and specificity.

\section{What this paper contributes to our knowledge}

Transverse scans at the level of the pharyngeal prominence and hyoid bone using a linear array transducer improved visualization of pharyngeal residue in the pyriform sinus and epiglottic vallecula. We observed an association between the presence of high-echogenicity areas in ultrasound images and the presence of pharyngeal residue as assessed with fiberoptic endoscopic swallowing evaluations, which is the standard method.

Real-time virtual sonography (RVS) is a new method that uses magnetic navigation and computer software for the montage display of real-time ultrasound and images from multiplanar reconstruction in magnetic resonance imaging (MRI). RVS has been used for diagnosis, treatment, and follow-up of patients with carcinomas. ${ }^{17-19}$ Studies have identified the hypopharyngeal area on MRIs ${ }^{20,21}$; therefore, we hypothesized that RVS could be used to visualize the pyriform sinus and the epiglottic vallecula to establish a method to scan for pharyngeal residue. Because RVS can display ultrasound images simultaneously with the corresponding MRIs in real time, the use of RVS will assist the detection of the pyriform sinus and epiglottic vallecula from ultrasound images, and it will show appropriate transducer position for the scanning.

RVS can identify the pyriform sinus and epiglottic vallecula in ultrasound images, but the technique will not be useful for individuals with dysphagia because MRI scanning requires individuals to keep still for extended periods of time. We therefore decided to use RVS data from individuals without dysphagia to evaluate the scanning method of the pyriform sinus and the epiglottic vallecula by ultrasound examination. In addition, we used ultrasound data obtained simultaneously from individuals with dysphagia who underwent FEES, which is the accepted method to evaluate pharyngeal residue. We considered that if there is no change of the structure in the oropharyngeal area, the results from healthy participants could be generalized to individuals with dysphagia.

We had two goals in this study. We aimed to systematize a methodology (ie, identify anatomical landmarks) 
for ultrasound evaluation of pharyngeal residue both in the pyriform sinus and epiglottic vallecula using RVS on healthy individuals. We sought to compare established ultrasound methodology for the detection of pharyngeal residue with the FEES approach performed at the same time in individuals with dysphagia.

\section{Methods}

Two cross-sectional observational studies were conducted to achieve our goals. The first part of this study evaluated the scanning method to detect the pyriform sinus and the epiglottic vallecula using RVS data from healthy individuals without dysphagia. The second evaluated the performance of ultrasound images obtained simultaneously with FEES from individuals with dysphagia to detect pharyngeal residue in the pyriform sinus and the epiglottic vallecula. The first part of this study was a preliminary phase that aimed to define the optimal position of the scan and transducer in the second part of the study. The ethics committee of our institution approved the study protocol (\#38-3). We obtained written informed consents from all subjects or their proxies.

\section{Subjects and Settings}

We collected all data for the first part of our study at the laboratory with RVS and MRI systems in June 2018. We included healthy subjects without dysphagia or history of laryngectomy or glossectomy. Next, we collected ultrasound and FEES data at 2 acute care hospitals from June 2018 to October 2018. We included individuals $>20$ y old who underwent FEES. Exclusion criteria included subjects with a neck area that made ultrasound scanning difficult, subjects who had difficulty following directions during examinations, and subjects with a history of laryngectomy or glossectomy.

\section{MRI Protocol for RVS Measurement}

We used a multiposture MRI system composed of a pair of $0.4 \mathrm{~T}$ permanent magnets that were opposed to each other (Hitachi Medical Corporation, Tokyo, Japan) 22 with a neck coil as a receiver. We took median sagittal and coronal plane images with static sequences while subjects were in the sitting position. We instructed the individuals to stop swallowing saliva and moving the tongue to avoid artifacts during imaging. We obtained $\mathrm{T}_{1}$-weighted transverse images with the following parameters: repetition time, $28 \mathrm{~ms}$; echo time, $18.4 \mathrm{~ms}$; flip angle, $18^{\circ}$; slice thickness, $2 \mathrm{~mm}$; acquisition matrix, $320 \times 224$ (readout $\times$ phase encoding); number of signals averaged, 1 ; receiver bandwidth, $\pm 26.0 \mathrm{kHz}$; and field of view, $200 \mathrm{~mm}$. An experienced radiologist confirmed that the pyriform sinus and epiglottic vallecula were visible in the images.

\section{RVS Protocol for the Investigation of the Scanning Method}

We loaded the digital imaging communications in the medicine MRI dataset into the RVS fusion system (ARIETTA 850, Hitachi, Tokyo, Japan) with a 5-18 MHz linear array transducer and displayed together with the ultrasound image on the same monitor. The scanning was conducted in a sitting position during the RVS protocol. Co-registration was performed using the laryngeal prominence as a reference, with a transducer attached vertically to the skin surface in a transverse scan. We used line markers included in the fusion system to identify the pyriform sinus and epiglottic vallecula. The ultrasonographic appearance and anatomic location of the pyriform sinus and the epiglottic vallecula were described. The position of the transducer was recorded using a digital camera when the pyriform sinus and epiglottic vallecula were visible in the ultrasound image.

\section{Ultrasound Protocol for Evaluating Performance for Pharyngeal Residue Detection}

We performed ultrasound examinations simultaneously with FEES for all subjects with dysphagia. The test liquid boluses included liquid with or without food thickener. The test food boluses included food with or without diet modification for subjects with dysphagia. For the test food with diet modification, we used 4 types of forms: jelly, puree, moist, and minced. All the test food and liquid boluses were modified based on the standard criteria of food texture in Japan, according to the Japanese Dysphagia Diet Criteria (JDD2013), ${ }^{23}$ defined by the Japanese Society of Dysphagia Rehabilitation depending on the individuals' swallowing function during the examination. Ultrasound examinations were performed with handheld ultrasonography devices (SonoSite iViz, Fujifilm, Tokyo, Japan) with 5-10 MHz or 6-13 MHz linear array transducers. The head and neck positions were not fixed to facilitate swallowing in the subjects' most comfortable posture. Subjects were placed in a semi-Fowler position with the head kept at $>30^{\circ}$ to prevent aspiration during the examination. We based the scanning method to visualize the pyriform sinus and epiglottic vallecula on the result of the RVS. The transducer was set at the level of the laryngeal prominence in the transverse direction of the left and right sides of the neck to visualize the left or right pyriform sinus (Fig. 1). The thyroid cartilage in the ultrasound image was used to identify the pyriform sinus. The transducer was set above the hyoid bone in the transverse direction to visualize the epiglottic vallecula (Fig. 2). The tongue base and the epiglottis in the ultrasound image were used to identify the epiglottic vallecula. A well-trained nurse with $>5 \mathrm{y}$ 

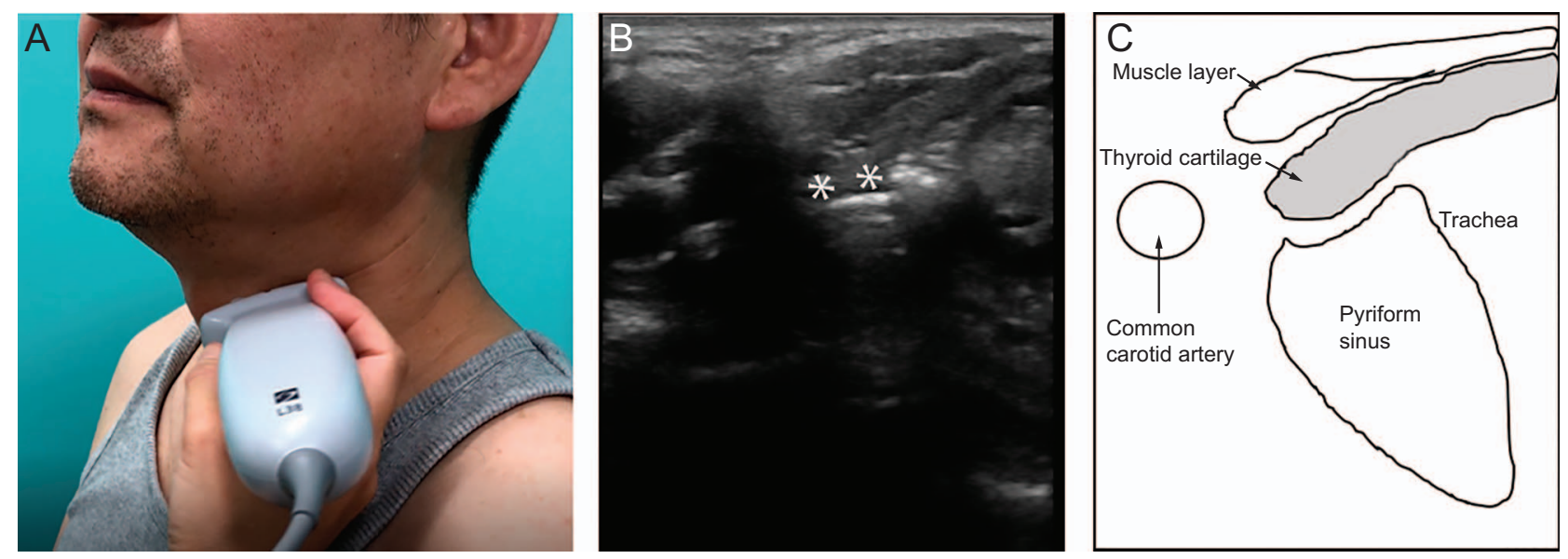

Fig. 1. A: Transducer position. B: Representative ultrasound image using the handheld device to detect pyriform sinus. C: Schema of the ultrasound image. Asterisks represent the air-mucosa interface.
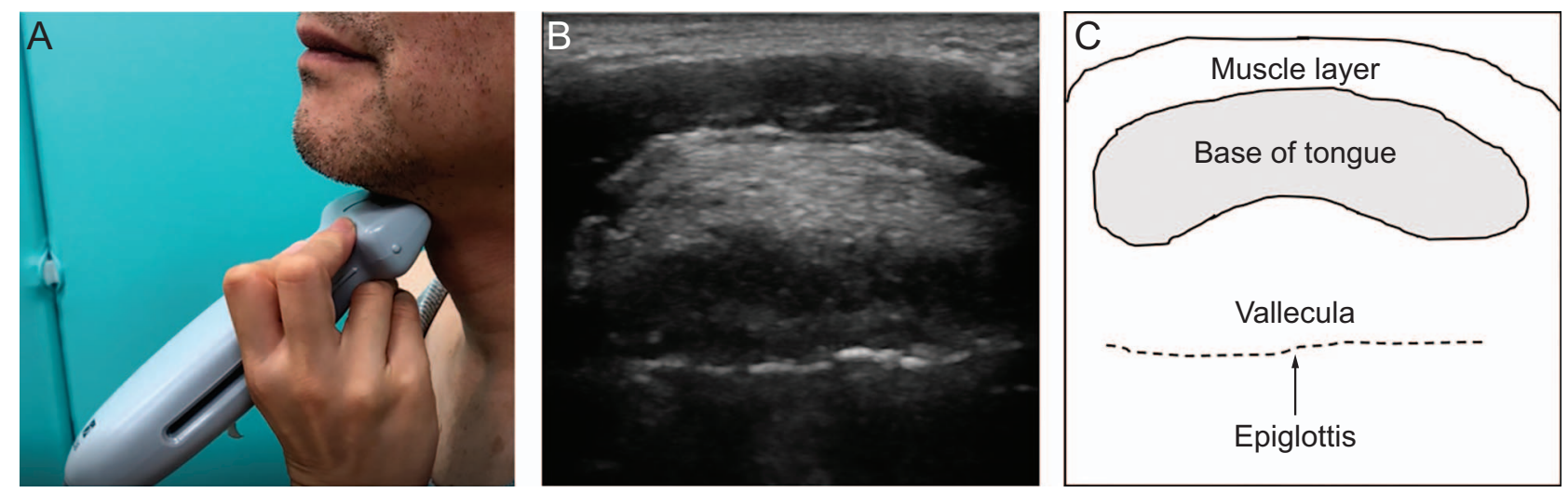

Fig. 2. A: Transducer position. B: Representative ultrasound image using the handheld device to detect epiglottic vallecula. C: Schema of the ultrasound image.

of experience in performing ultrasound examinations conducted all examinations. The operator obtained ultrasound videos pre- or post-swallowing of $6-15 \mathrm{~s}$ at a rate of 30 frames/s.

The videos were converted to $180-450$ consecutive images. Next, all images showing clear pyriform sinus and epiglottic vallecula were selected. Images that did not show these landmarks were excluded from the analysis. After the selection of images of the best quality, we measured the areas of the pyriform sinus, the epiglottic vallecula, and a high-echogenicity area in these regions using ImageJ software (National Institutes of Health, Bethesda, MD) (Figs. 3 and 4). A high-echogenicity area was defined as a region that could be distinguished from surroundings due to its high echogenicity. We also measured the proportion of the high-echogenicity area to the pyriform sinus and epiglottic vallecula areas. These measurements were performed by a researcher who was blinded to the FEES results.

\section{FEES Protocol as a Reference Method for Pharyngeal Residue Detection}

All subjects received FEES performed by experienced dentists simultaneously with ultrasound examinations. Subjects were positioned as during usual mealtimes, and a fiberscope (ENF TYPE P4 or V2; Olympus, Tokyo, Japan) was inserted through the nose. Water-based lubricant without anesthetic was applied to the tip of the fiberscope to enhance the exam tolerability and to avoid impairing the swallowing ability. The dentists decided on the order of the test food and liquid boluses and the number of measurements to be performed, and they also evaluated the level of pharyngeal residue for both the pyriform sinus and the epiglottic vallecula. We used a published classification for the level of pharyngeal residue: none, no visible boluses or secretions; mild, up to half of the pyriform sinus or epiglottic vallecula covered with boluses or secretions; and severe, more than half of the pyriform sinus or epi- 

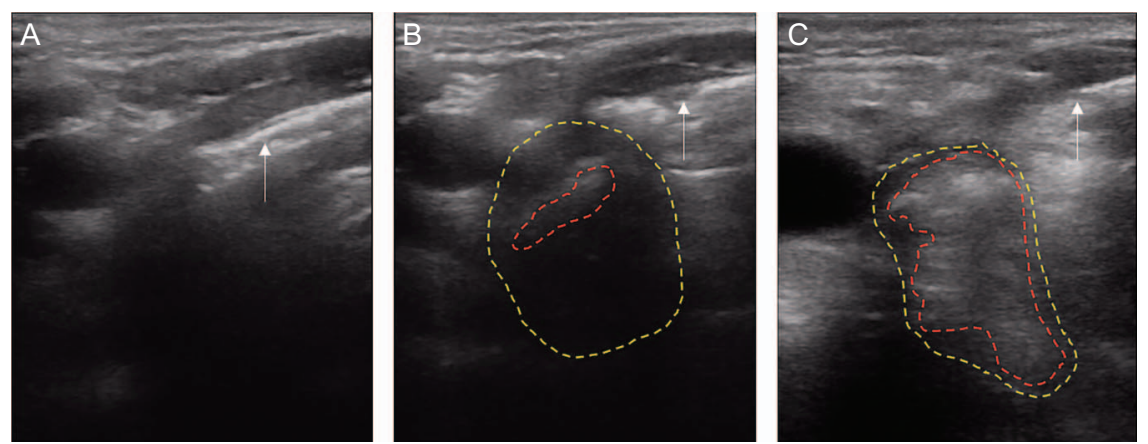

Fig. 3. Examples of the measurements of high-echogenicity areas in ultrasound images of the pyriform sinus. A: $0 \%, \mathrm{~B}: 10.9 \%, \mathrm{C}: 80.0 \%$. The yellow dashed lines represent the pyriform sinuses. The red dashed lines represent high-echogenicity areas. Arrows indicate the thyroid cartilage.
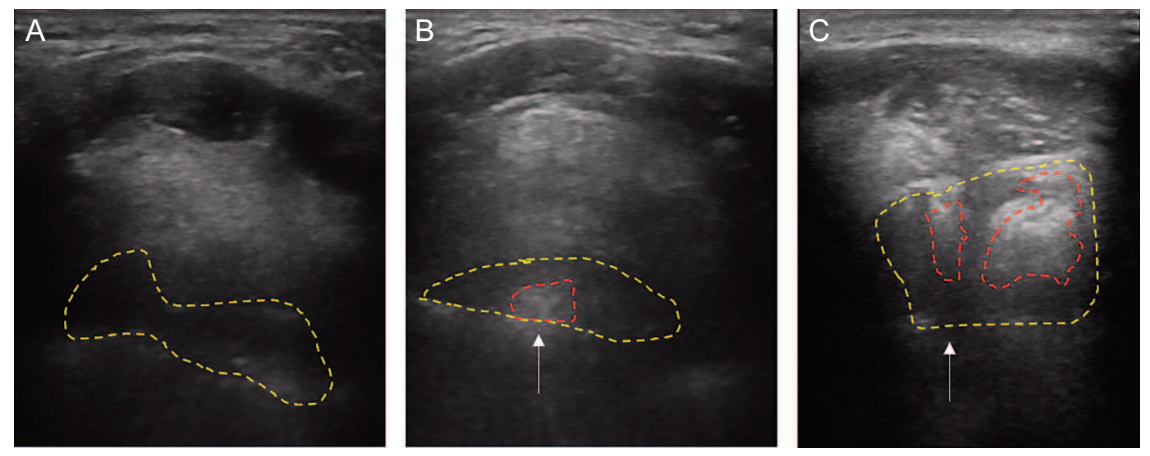

Fig. 4. Examples of the measurements of high-echogenicity areas in the ultrasound images of the epiglottic vallecula. A: $0 \%, \mathrm{~B}: 22.3 \%$, C: $42.5 \%$. The yellow dashed lines represent the epiglottic vallecula. The red dashed lines represent high-echogenicity areas. Arrows indicate the epiglottis.
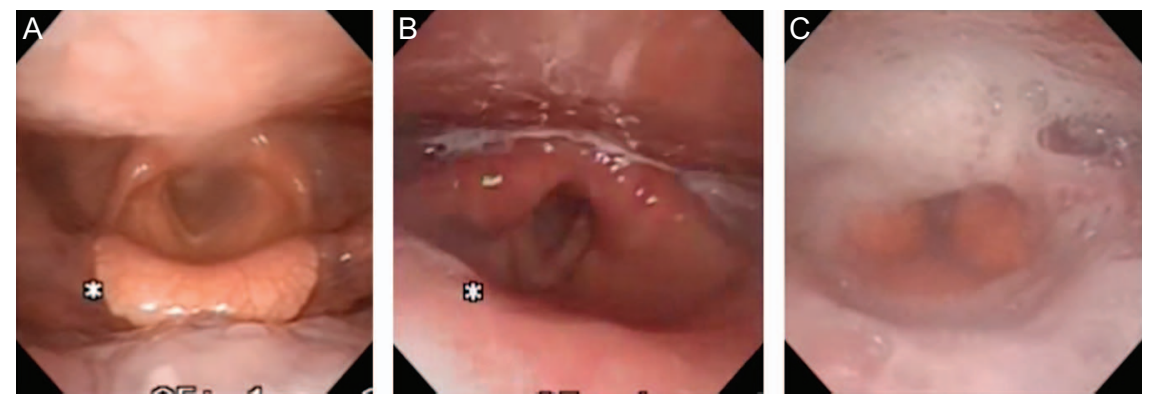

Fig. 5. Pyriform sinus images produced with fiberoptic endoscopic evaluation of swallowing (FEES) according to the pharyngeal residue classification. A: None, B: mild, and C: severe. Asterisks are markers of the fiberscope system to adjust focus in the image.

glottic vallecula covered with boluses or secretions (Figs. 5 and 6). ${ }^{24}$

\section{Data Analysis}

We expressed descriptive data as mean \pm SD for continuous variables and as $n(\%)$ for categorical variables. To assess whether the proportion of the high-echogenicity area to the pyriform sinus and the epiglottic vallecula areas can be used as a marker for pharyngeal residue detection, we used a receiver operating characteristic (ROC) curve. We defined any mild and severe level of pharyngeal residue detected by FEES as the presence of pharyngeal residue. We measured the area under the curve and calculated the sensitivity, specificity, and positive and negative predictive values at 4 cutoff points $(0$, $0.05,0.1$, and 0.5$)$. We set the $P$ value for statistical significance at .05 for each analysis. All analyses were conducted using STATA 14 software (STATA, College Station, Texas). 

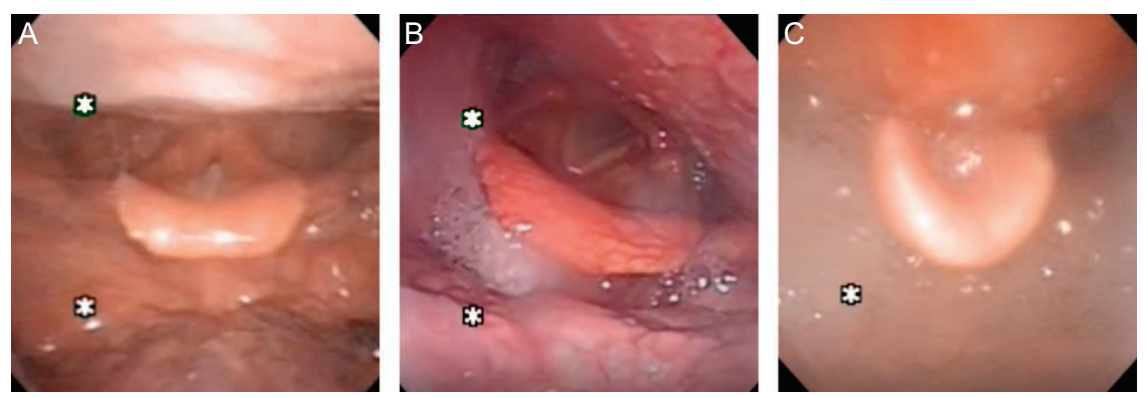

Fig. 6. Epiglottic vallecula images produced with fiberoptic endoscopic evaluation of swallowing (FEES) according to the pharyngeal residue classification. A: None, B: mild, and C: severe. Asterisks are markers of the fiberscope system to adjust focus in the image.
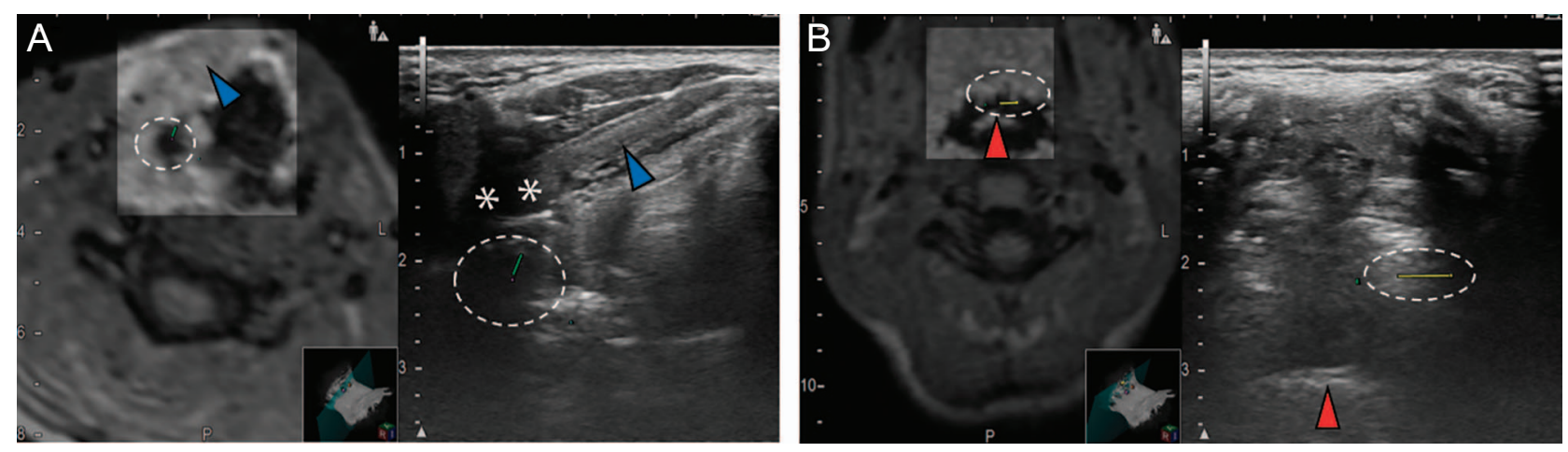

Fig. 7. Representative images using real-time virtual sonography, which is a fusion of magnetic resonance imaging and ultrasound imaging, to detect A: the pyriform sinus and B: the epiglottic vallecula. Lines in the dashed circles were used to identify the pyriform sinus and the epiglottic vallecula. Blue arrows indicate the thyroid cartilage, asterisks show the air-mucosa interface, and red arrows indicate epiglottis.

\section{Results}

Four healthy subjects ( 3 men, 1 woman) $20-50$ y old participated in the first part of the study to assess the scanning method to detect the pyriform sinus and epiglottic vallecula by RVS. The locations of the pyriform sinus and epiglottic vallecula marked by the fusion system were detected from ultrasound images of all the individuals. The pyriform sinus with low echogenicity was located below the thyroid cartilage (Fig. 7A). The air-mucosa interface around the pyriform sinus appeared as a high-echogenicity line. Once the pyriform sinus was visible in ultrasound images, the transducer was located at the level of laryngeal prominence in the transverse direction. The epiglottic vallecula with low echogenicity was located between the tongue base and the epiglottis, which were visible as high echogenic regions and lines (Fig. 7B). Once the epiglottic vallecula was visible in the ultrasound image, the transducer was located above the hyoid bone in a transversal direction.

Thirty-seven subjects who underwent FEES were assessed for eligibility before recruitment to the study that aimed to investigate the performance of ultrasound for detecting pharyngeal residue. We obtained written informed consents from 36 individuals or their proxies. We excluded one individual with tracheotomy from the study. No individuals had difficulty following directions or swallowing due to the attachment of the transducer during the ultrasound examinations. Finally, we included data from 35 subjects with dysphagia ( 26 men, 9 women, $80.4 \pm 10.6$ y old) in the analysis.

Table 1 details the characteristics of the 35 subjects enrolled. Fourteen subjects had cognitive impairment, and $25(71.4 \%)$ were diagnosed with pharyngeal residue in the pyriform sinus and epiglottic vallecula by FEES. However, 4 subjects $(11.4 \%)$ had residue only in the pyriform sinus, and 6 showed no residue with FEES.

We obtained 245 ultrasound images for assessment of residue in the pyriform sinus from 35 subjects and $85 \mathrm{ul}-$ trasound images for assessment of residue in the epiglottic vallecula from 26 subjects who underwent FEES simultaneously. We could not obtain ultrasound images of the epiglottic vallecula from 9 subjects because scanning above the hyoid bone was difficult due to severe contractures of the neck, difficulty moving the head, or the presence of gauze dressings to cover a stoma for a mini-tracheostomy. After excluding 7 images and $3 \mathrm{im}-$ ages in which the pyriform sinus and the epiglottic vallecula were not distinguishable, respectively; we used 238 and 82 ultrasound images for analyses to assess the 
Table 1. Characteristic of the Enrolled Individuals

\begin{tabular}{lc}
\hline \multicolumn{1}{c}{ Characteristics } & Result \\
\hline Age, y, mean \pm SD & $80.4 \pm 10.6$ \\
Men & $26(74.3)$ \\
Main disease associated with dysphagia & \\
Pneumonia & $15(42.9)$ \\
Stroke & $13(37.1)$ \\
Cerebral hemorrhage & $2(5.7)$ \\
Cervical spine injury & $1(2.9)$ \\
Others & $4(11.4)$ \\
Cognitive impairment & $14(40.0)$ \\
The Food Intake LEVEL Scale & $16(45.7)$ \\
Level 1-3: no oral intake & $4(11.4)$ \\
Level 4-6: oral intake and alternative nutrition & $15(42.9)$ \\
Level 7-9: oral intake alone & $4(11.4)$ \\
Use of tube feeding & $23(65.7)$ \\
Presence of cough during eating and swallowing & \\
Results of aspiration during FEES & $11(31.4)$ \\
Presence of aspiration & $24(68.6)$ \\
No aspiration & \\
Results of pharyngeal residue during FEES & $25(71.4)$ \\
Presence of residue in pyriform sinus and vallecula \\
Presence of residue in pyriform sinus only \\
No residue in pyriform sinus and vallecula \\
\hline Data are presented as $n$ (\%) unless otherwise noted. \\
FEES = fiberoptic endoscopic evaluation of swallowing \\
\hline
\end{tabular}

performance of ultrasound examination in detecting pharyngeal residue. In total, pharyngeal residue was detected in 174 ultrasound images of the pyriform sinus and 60 ultrasound images of the epiglottic vallecula with reference to the FEES. From these totals, pharyngeal residue was the result of secretions in 98 pyriform sinus and 22 epiglottic vallecula images.

The receiver operating characteristic curve showed that the proportion of high-echogenicity area was an accurate marker of pharyngeal residue positivity; the areas under the curve were 0.88 (95\% CI 0.83-0.93) (Figure 8A) for residue in the pyriform sinus and 0.87 (95\% CI 0.79-0.95; Fig. 8B) for residue in the epiglottic vallecula. Tables 2 and 3 show the sensitivity, specificity, and positive and negative predictive values for detection of pharyngeal residue according to the threshold level of the proportion of high-echogenicity area at the cutoff points of $0,0.05,0.1$, and 0.5 . The sensitivity and specificity for the detection of pharyngeal residue in the pyriform sinus were $92.0 \%$ and $71.9 \%$, respectively, when the threshold proportion of highechogenicity area was set at 0 . The sensitivity and specificity for the detection of pharyngeal residue in the epiglottic vallecula were $86.7 \%$ and $63.6 \%$, respectively, when the threshold proportion of high-echogenicity area was set at 0 .
The residue of liquid without food thickener in the pyriform sinus and residue of all the tested liquid boluses (with or without food thickener) and the 4 tested food boluses in the epiglottic vallecula showed significant high echogenicity in ultrasound images. However, 2 of the 5 liquids with food thickener, 4 of 13 jelly, 2 of 14 pureed food, 2 of 18 moist food, 1 of 13 minced food, and 3 of 98 secretions did not show any high echogenicity in ultrasound images of the pyriform sinus. Eight of 22 secretions did not show any high echogenicity in ultrasound images of the epiglottic valley.

\section{Discussion}

One of the main findings of this study was that we identified anatomical landmarks to systematize a methodology for ultrasound evaluation of pharyngeal residue in both the pyriform sinus and the epiglottic vallecula with RVS. A montage of MRI and ultrasound images showed that the thyroid cartilage, the tongue base, and the epiglottis were useful as landmarks for detecting the pyriform sinus and the epiglottic vallecula in a transversal scan. Images from this fusion system also showed the adequate transducer position for the scanning. To visualize the pyriform sinus, the transducer needs to be positioned on the level of the laryngeal prominence, and to visualize the epiglottic vallecula, the transducer needs to be above the hyoid bone.

We have established a methodology for the ultrasound evaluation of the presence of pharyngeal residue. In a previous study, ${ }^{15}$ we obtained images with longitudinal scans, but the attenuation of the ultrasound beam due to air in the trachea often occurred, resulting in a sensitivity and specificity of $62 \%$ and $67 \%$, respectively, for detecting pharyngeal residue. The method described in this study, which is based on a transversal scan, allows the visualization of the pyriform sinus and epiglottic vallecula while bypassing the trachea and its problematic volume of air. This improved the quality of images of the pyriform sinus, the epiglottic vallecula, and the residue in these areas, leading to a sensitivity and specificity of $92.0 \%$ and $71.9 \%$, respectively, for the detection of pharyngeal residue in the pyriform sinus.

The best results for the detection of pharyngeal residue occurred when the threshold proportion of high-echogenicity area was set at $5 \%$. High sensitivity is required for a screening test that aims to detect insufficient pharyngeal clearance and to remove the pharyngeal residue. Because the sensitivities are $>80 \%$ at the cutoff point of $0 \%$, we recommend using the presence of high-echogenicity areas as markers of abnormal findings in the pyriform sinus and epiglottic vallecula in clinical settings. The sensitivity of pharyngeal residue detection with our method was higher than that found in the previous study $(62 \%)^{15}$ when the 

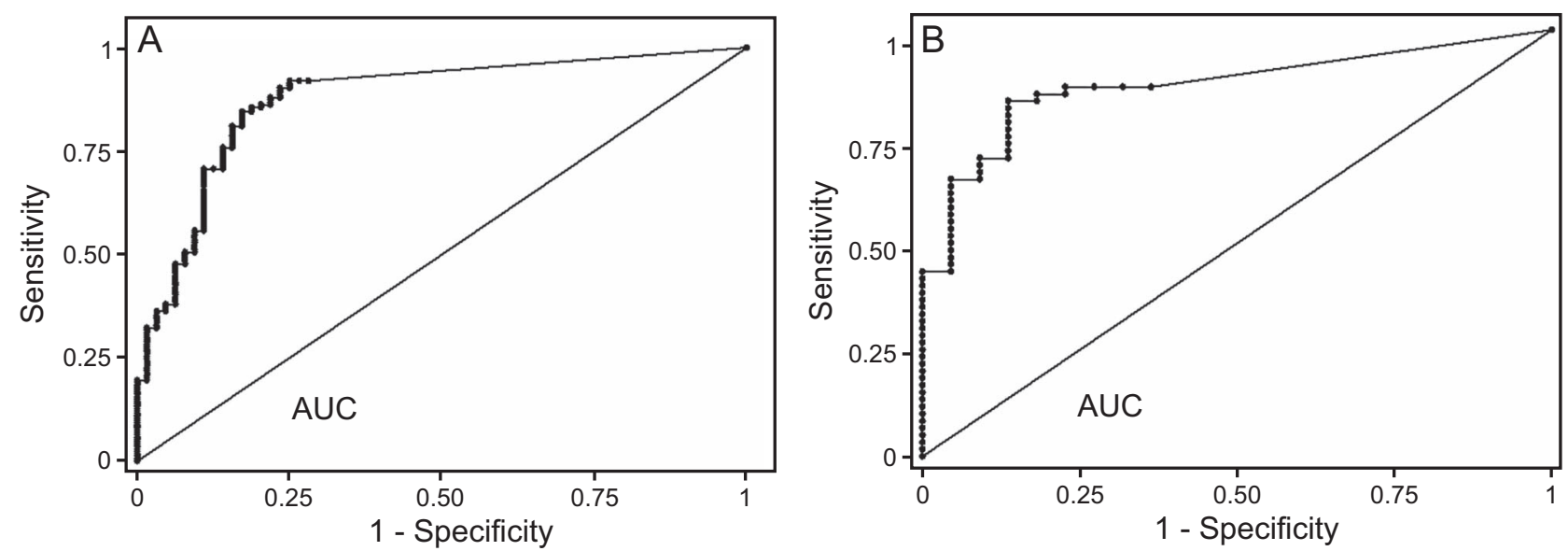

Fig. 8. Receiver operating characteristic curve for the proportion of high-echogenicity areas for detecting pharyngeal residue in A: the pyriform sinus and B: the epiglottic vallecula. AUC = area under the curve.

Table 2. Performance of the Method for Detection of Pharyngeal Residue in the Pyriform Sinus Based on 4 Cutoff Points

\begin{tabular}{|c|c|c|c|c|}
\hline $\begin{array}{l}\text { Proportion of High } \\
\text { Echogenicity Area }\end{array}$ & Sensitivity & Specificity & $\begin{array}{c}\text { Positive Predictive } \\
\text { Value }\end{array}$ & $\begin{array}{c}\text { Negative Predictive } \\
\text { Value }\end{array}$ \\
\hline$<0$ & $92.0(86.9-95.5)$ & $71.9(59.2-82.4)$ & $89.9(84.4-93.9)$ & $76.7(64.0-86.6)$ \\
\hline$\leq 0.05$ & $87.9(82.1-92.4)$ & $78.1(66.0-87.5)$ & $91.6(86.3-95.3)$ & $70.4(58.4-80.7)$ \\
\hline$\leq 0.1$ & $79.3(72.5-85.1)$ & $84.4(73.1-92.2)$ & $93.2(87.9-96.7)$ & $60.0(49.1-70.2)$ \\
\hline$\leq 0.5$ & $21.3(15.4-28.1)$ & $98.4(91.6-100.0)$ & $97.4(86.2-99.9)$ & $31.5(25.1-38.4)$ \\
\hline
\end{tabular}

$\overline{\text { Data are presented as }} \%(95 \% \mathrm{CI})$.

Table 3. Performance of the Method for Detection of Pharyngeal Residue in the Vallecula Based on 4 Cutoff Points

\begin{tabular}{|c|c|c|c|c|}
\hline $\begin{array}{l}\text { Proportion of High } \\
\text { Echogenicity Area }\end{array}$ & Sensitivity & Specificity & $\begin{array}{c}\text { Positive Predictive } \\
\text { Value }\end{array}$ & $\begin{array}{c}\text { Negative Predictive } \\
\text { Value }\end{array}$ \\
\hline$<0$ & $86.7(75.4-94.1)$ & $63.6(40.7-82.8)$ & $86.7(75.4-94.1)$ & $63.6(40.7-82.8)$ \\
\hline$\leq 0.05$ & $85.0(73.4-92.9)$ & $81.8(59.7-94.8)$ & $92.7(82.4-98.0)$ & $66.7(46.0-83.5)$ \\
\hline$\leq 0.1$ & $75.0(62.1-85.3)$ & $86.4(65.1-97.1)$ & 93.8 (82.8-98.7) & $55.9(37.9-72.8)$ \\
\hline$\leq 0.5$ & $5.0(1.0-13.9)$ & $100.0(84.6-100.0)$ & $100.0(29.2-100.0)$ & $27.9(18.3-39.1)$ \\
\hline
\end{tabular}

$\overline{\text { Data are presented as }} \%(95 \% \mathrm{CI})$.

proportion of high-echogenicity area was set at $10 \%$. In case of failure to detect a small amount of residue, our new scanning method should perform better. If ultrasound examination reveals high-echogenicity areas in the pyriform sinus or epiglottic vallecula, the patient will probably require residue removal to prevent aspiration pneumonia.

The extent of high echogenicity identified on ultrasound images varied among the types of residues. Some boluses did not produce significant high echogenicity. The sensitivity for the detection of pharyngeal residue of liquid without food thickener and jelly in the pyriform sinus and secretions in the epiglottic valley were $<70 \%$. The reason is related to the difference of the volume of the residue or the amount of air that exists as bubbles in the test foods. The small amount of the residue and homogenized boluses without bubbles made it difficult to produce high echogenicity because there are few materials that cause ultrasound reflection. ${ }^{25}$

For clinical implementation, noninvasive and real-time ultrasound examinations for the assessment of pharyngeal residue will facilitate secretion management and pharyngeal clearance. In this study, more than half of the cases of residue in the pyriform sinus were due to secretions. This suggests that secretion management is essential for individuals with dysphagia in addition to swallowing care. If high echogenicity is detected with ultrasound examination 
in the pyriform sinus before using test foods or liquids, pharyngeal clearance will be insufficient because of the presence of secretions. Such patients may silently aspirate secretions and are at a high risk for aspiration pneumonia. ${ }^{26}$ Suctioning is needed to prevent aspiration pneumonia in these cases. We propose ultrasound assessment of pharyngeal residue before and during mealtimes. The positioning of the transducer on the neck during pre- and post-swallowing was well tolerated by subjects, and we were able to see the pyriform sinus in all subjects. However, for some individuals, the visualization of the epiglottic vallecula was impossible because of the difficulty to position the transducer on the narrow area between the neck and the chin. A smaller transducer should be developed for these cases.

The main limitation of this study is that the ultrasound examinations were performed by a single experienced nurse. Because we aimed to investigate the performance of detecting pharyngeal residue with ultrasound examination according to a new method, we limited our design to a single operator with high scanning and observation skills. Structured training programs will be needed to teach the skills for ultrasound imaging and the observation methods for detecting pharyngeal residue. Future studies are needed to evaluate the reproducibility of our results by nurses who complete training programs for ultrasound examination using this method. Moreover, image-processing methods that automatically color regions of aspirated boluses in ultrasound images have contributed to improvement for performance of aspirated-bolus detection. ${ }^{27}$ It was difficult to establish a standard of semiquantitative classification of residue in ultrasound images in this study because we did not know whether the size of the high-echogenicity areas in ultrasound images correlated with the amount of pharyngeal residue. Future studies to investigate the relationship between the level of the pharyngeal residue and the size of the high echogenicity is needed for a semiquantitative evaluation. We believe that our image processing method will be useful for validated pharyngeal residue evaluation in the pyriform sinus and the epiglottic vallecula.

Another limitation of this study is that the first part of the study, which aimed to define the optimal position of the scan and transducer, used data from only 4 healthy subjects. We excluded individuals with a history of laryngectomy or glossectomy; therefore, the results were generalized to individuals with dysphagia. We need to confirm the applicability of the scanning method for the individuals with structural changes in the oropharyngeal area in the next step.

\section{Conclusions}

The transverse ultrasound scan above the thyroid cartilage and hyoid bone enabled visualization of the pyriform sinus, epiglottic vallecula, and pharyngeal residue. The presence of high-echogenicity areas resulted in a sensitivity of $92.0 \%$ and a specificity of $71.9 \%$ for detecting pharyngeal residue in the pyriform sinus and a sensitivity of $86.7 \%$ and specificity of $63.6 \%$ for detecting pharyngeal residue in the epiglottic vallecula.

\section{ACKNOWLEDGMENTS}

We thank Dr Toshiaki Miyati and Dr Naoki Ohno, who provided advice and helped with the collection of MRI data. We thank Dr Takashi Hase and Dr Nario Matsumoto, who provided advice and helped with the collection of FEES data from individuals with dysphagia.

\section{REFERENCES}

1. Murray J, Langmore SE, Ginsberg S, Dostie A. The significance of accumulated oropharyngeal secretions and swallowing frequency in predicting aspiration. Dysphagia 1996;11(2):99-103.

2. Kelly AM, Macfarlane K, Ghufoor K, Drinnan MJ, Lew-Gor S. Pharyngeal residue across the lifespan: a first look at what's normal. Clin Otolaryngol 2008;33(4):348-351.

3. Shapira-Galitz Y, Shoffel-Havakuk H, Halperin D, Lahav MY. Correlation between pharyngeal residue and aspiration in fiberoptic endoscopic evaluation of swallowing - an observational study. Arch Phys Med Rehabil 2019;100(3):488-494.

4. Han TR, Paik NJ, Park JW. Quantifying swallowing function after stroke: a functional dysphagia scale based on videofluoroscopic studies. Arch Phys Med Rehabil 2001;82(5):677-682.

5. Morimoto K, Suzuki M, Ishifuji T, Yaegashi M, Asoh N, Hamashige $\mathrm{N}$, et al. The burden and etiology of community-onset pneumonia in the aging Japanese population: a multicenter prospective study. PLoS One 2015;10(3):e0122247.

6. Park BH, Seo JH, Ko MH, Park SH. Effect of 45 degrees reclining sitting posture on swallowing in patients with dysphagia. Yonsei Med J 2013;54(5):1137-1142.

7. Steele CM, Alsanei WA, Ayanikalath S, Barbon CE, Chen J, Cichero JA, et al. The influence of food texture and liquid consistency modification on swallowing physiology and function: a systematic review. Dysphagia 2015;30(1):2-26.

8. Inui Y, Kamakura Y, Fukada J, Yoneda M, Kataoka E, Usami Y, et al. Development of pyriform sinus suctioning programs for aspiration pneumonia prevention during the acute stroke. Dysphagia 2017; 32(6):767-776.

9. Pearson WG Jr., Molfenter SM, Smith ZM, Steele CM. Image-based measurement of post-swallow residue: the normalized residue ratio scale. Dysphagia 2013;28(2):167-177.

10. Leder SB, Murray JT. Fiberoptic endoscopic evaluation of swallowing. Phys Medicine Rehabil Clin N Am 2008;19(4):787-801.

11. Yamashita M, Yokoyama K, Takei Y, Furuya N, Nakamichi Y, Ihara $\mathrm{Y}$, et al. Acoustic characteristics of voluntary expiratory sounds after swallow for detecting dysphagia. J Oral Rehabil 2014;41(9):667674.

12. Lagarde ML, Kamalski DM, van den Engel-Hoek L. The reliability and validity of cervical auscultation in the diagnosis of dysphagia: a systematic review. Clin Rehabil 2016;30(2):199-207.

13. Adhikari S, Zeger W, Schmier C, Crum T, Craven A, Frrokaj I, et al. Pilot study to determine the utility of point-of-care ultrasound in the assessment of difficult laryngoscopy. Acad Energ Med 2011;18(7): 754-758.

14. Reddy PB, Punetha P, Chalam KS. Ultrasonography: a viable tool for airway assessment. Indian J Anaesth 2016;60(11):807-813. 


\section{Detecting Pharyngeal Residue by Ultrasonography}

15. Miura Y, Nakagami G, Yabunaka K, Tohara H, Hara K, Noguchi $\mathrm{H}$, et al. Detecting pharyngeal post-swallow residue by ultrasound examination: a case series. Med Ultrason 2016;18(3):288-293.

16. Miura Y, Nakagami G, Yabunaka K, Tohara H, Murayama R, Noguchi $\mathrm{H}$, et al. Method for detection of aspiration based on B-mode video ultrasonography. Radiol Phys Technol 2014;7(2): 290-295.

17. Uematsu T, Takahashi K, Nishimura S, Watanabe J, Yamasaki S, Sugino $\mathrm{T}$, et al. Real-time virtual sonography examination and biopsy for suspicious breast lesions identified on MRI alone. Eur Radiol 2016;26(4):1064-1072.

18. Nakano S, Yoshida M, Fujii K, Yorozuya K, Kousaka J, Mouri Y, et al. Real-time virtual sonography, a coordinated sonography and MRI system that uses magnetic navigation, improves the sonographic identification of enhancing lesions on breast MRI. Ultrasound Med Biol 2012;38(1):42-49.

19. Lee CH, Chen WT, Lin CC, Teng W, Lin SM, Chiu CT. Radiofrequency ablation assisted by real-time virtual sonography for hepatocellular carcinoma inconspicuous under sonography and high-risk locations. Kaohsiung J Med Sci 2015;31(8):413-419.

20. Honda Y, Hata N. Dynamic imaging of swallowing in a seated position using open-configuration MRI. J Magn Reson Imaging 2007; 26(1):172-176.

21. Bradley JA, Paulson ES, Ahunbay E, Schultz C, Li XA, Wang D. Dynamic MRI analysis of tumor and organ motion during rest and deglutition and margin assessment for radiotherapy of headand-neck cancer. Int J Radiat Oncol Biol Phys 2011;81(5): e803-e812.

22. Kadoya Y, Miyati T, Kobayashi S, Ohno N, Gabata T. Effect of gravity on portal venous flow: evaluation using multiposture MRI. J Magn Reson Imaging 2019;50(1):83-87.

23. Shimizu A, Maeda K, Tanaka K, Ogawa M, Kayashita J. Texturemodified diets are associated with decreased muscle mass in older adults admitted to a rehabilitation ward. Geriatr Gerontol Int 2018; 18(5):698-704

24. Neubauer PD, Rademaker AW, Leder SB. The Yale Pharyngeal Residue Severity Rating Scale: an anatomically defined and imagebased tool. Dysphagia 2015;30(5):521-528.

25. Calliada F, Campani R, Bottinelli O, Bozzini A, Sommaruga MG. Ultrasound contrast agents: basic principles. Eur J Radiol 1998; 27(Suppl 2):S157-S160.

26. Takahashi N, Kikutani T, Tamura F, Groher M, Kuboki T. Videoendoscopic assessment of swallowing function to predict the future incidence of pneumonia of the elderly. J Oral Rehabil 2012;39(6): 429-437.

27. Miura Y, Nakagami G, Yabunaka K, Tohara H, Murayama R, Noguchi $\mathrm{H}$, et al. Method for detecting aspiration based on image processing-assisted B-mode video ultrasonography. J Nurs Sci Eng 2014;1(1):12-20

This article is approved for Continuing Respiratory Care Education credit. For information and to obtain your CRCE

(free to AARC members) visit

www.rcjournal.com 